\title{
Direct machining of curved trenches in silicon with femtosecond accelerating beams
}

\section{A. Mathis \\ L. Froehly \\ L. Furfaro \\ M. Jacquot}

\section{J. M. Dudley}

\section{F. Courvoisier}

francois.courvoisier@femto-st.fr
Département d'Optique P.M. Duffieux, Institut FEMTO-ST, UMR 6174 CNRS Université de FrancheComté, 25030 Besançon Cedex, France

Département d'Optique P.M. Duffieux, Institut FEMTO-ST, UMR 6174 CNRS Université de FrancheComté, 25030 Besançon Cedex, France

Département d'Optique P.M. Duffieux, Institut FEMTO-ST, UMR 6174 CNRS Université de FrancheComté, 25030 Besançon Cedex, France

Département d'Optique P.M. Duffieux, Institut FEMTO-ST, UMR 6174 CNRS Université de FrancheComté, 25030 Besançon Cedex, France

Département d'Optique P.M. Duffieux, Institut FEMTO-ST, UMR 6174 CNRS Université de FrancheComté, 25030 Besançon Cedex, France

Département d'Optique P.M. Duffieux, Institut FEMTO-ST, UMR 6174 CNRS Université de FrancheComté, 25030 Besançon Cedex, France

Control of the longitudinal profile of ablated structures during laser processing is a key technological requirement. We report here on the direct machining of trenches in silicon with circular profiles using femtosecond accelerating beams. We describe the ablation process based on an intensity threshold model, and show how the depth of the trenches can be predicted in the framework of a caustic description of the beam. [DOI: http://dx.doi.org/10.2971/jeos.2013.13019]

Keywords: Accelerating beams, airy beams, femtosecond laser micromachining

\section{INTRODUCTION}

In the technological context of wafer and glass panels dicing, edge profiles are often a key parameter. Indeed, their shape is determinant for stress residues, packaging, mechanical properties of the diced workpiece. In contrast with mechanical techniques, laser dicing offers many benefits in terms of processing speed since it is contact-free. However, the control on edge profile of ablated structures is challenging, particularly when processing at micrometric scales. Ablation with standard Gaussian beams generally leads to tapered trenches. Femtosecond Bessel beams allow micro- and nano- machining with vertical walls, but this approach is valid only for transparent materials [1, 2].

In contrast, accelerating beams provide a novel degree of control of the longitudinal profile of ablated structures. Accelerating beams are solutions to the wave equation whose intensity peak travels along a curved trajectory during the propagation, and exhibit a transverse profile close to an Airy function [3]. These beams attracted much attention in the field of optics, particle manipulation and nonlinear optics $[4,5]$, but it is only recently that nonparaxial beams were theoretically and experimentally demonstrated [6]-[8]. In addition, arbitrary curved trajectories can be produced [9]-[13].

Accelerating beams possess a specific spatial distribution of light. Figure 1 shows a 3D view of the accelerating beam, where the red part shows the iso-intensity volume at half max- imum. This volume is approximately a bent cylinder while the remaining part of the beam, composed by several lobes of much lower intensity, propagates outside this trajectory. In the figure, the gold volume corresponds to the iso-intensity at $5 \%$ of the maximal intensity. Importantly, no light propagates inside the region delimited by the trajectory of the main intensity lobe. We recently demonstrated that this beam structure can be used as an efficient tool to process arbitrary convex

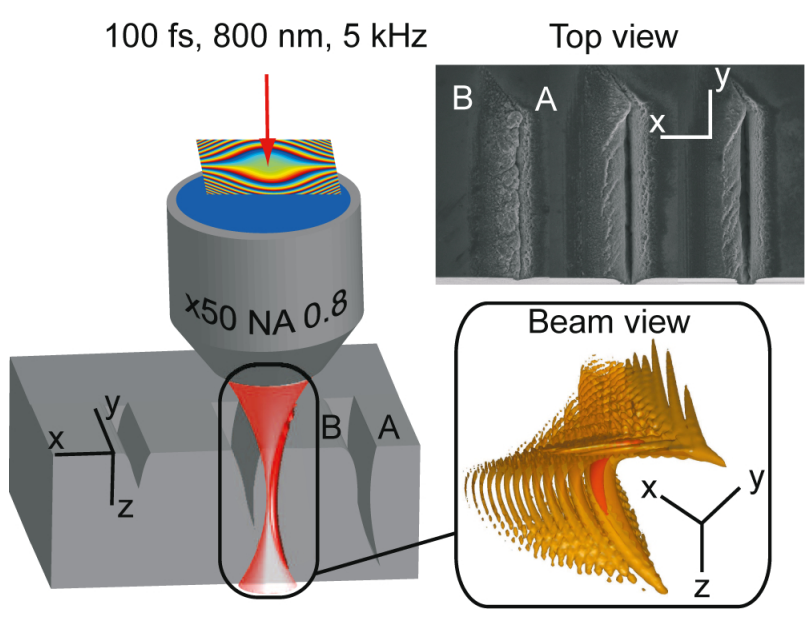

FIG. 1 Experimental setup used for laser machining of curved trenches in silicon. On the right side of the figure, we show a top view of a sample after processing and a $3 \mathrm{D}$ reconstruction of the beam (see text). 
profiles in transparent and opaque materials [14]. Two types of laser processing strategies can be developed. This first is edge profiling: the intense curved core contributes to ablation while the rest of the beam freely propagates in air. We showed in [14] that the final profile is determined from the fluence isocontour at the ablation threshold. The second strategy is using the accelerating beam directly incident upon the workpiece to process curved trenches, as shown in Figure 1. In this case, however, the ablation mechanism is not straightforward. The main objective here is to uncover this mechanism. We investigate the process of direct machining of curved trenches with accelerating beams and show that the beam structure is a key parameter to describe the drilling process and how it evolves with energy per pulse.

\section{EXPERIMENTAL SETUP}

Our experiments use the setup summarized in Figure 1. The beam of an amplified $5 \mathrm{kHz}$ Ti:Sa laser emitting $100 \mathrm{fs}$ pulses at a central wavelength of $800 \mathrm{~nm}$ is spatially shaped by an optically-addressed spatial light modulator (Hamamatsu PAL-SLM). The phase mask was designed in order to produce an accelerating beam in the first order of diffraction. Two lenses placed at confocal distances, realize an image of the SLM with a de-magnification factor of 3. Spatial filtering is used at the focal plane of the first lens to eliminate spurious diffraction orders. The SLM image is placed at the back focal plane of a high numerical aperture (NA 0.8) microscope objective as shown in Figure 1. The accelerating beam is produced in the focal region of the microscope objective.

Phase masks were designed by using a similar approach of the one described in references $[10,15]$. Here, we designed the phase mask so as to produce a beam propagating along a $120 \mu \mathrm{m}$ radius circular trajectory. In the vicinity of the main intensity lobe the beam can be well approximated by two dimensional Airy functions perpendicular to each other, i.e. $|A i(\alpha(x-y)) \cdot A i(\alpha(x+y))|^{2}$ with central lobe FWHM of $\sim 2 \mu \mathrm{m}[7]$.

Sample positioning is performed under white light illumination by visualization through the focusing microscope objective on a CCD camera as in reference [16]. The longitudinal positioning error between the accelerating beam and the sample is less than $3 \mu \mathrm{m}$. Samples were placed on a gimbals mount to ensure planarity better than $1 \mathrm{mrad}$ and were translated with respect to the beam by a motorized precision XYZ translation stage. We investigated trenching on $100 \mu \mathrm{m}$ thick silicon samples. After laser trench machining, the samples were mechanically diced and polished with a precision dicing saw and further imaged by scanning electron microscopy (SEM).

\section{CURVED TRENCH MACHINING}

Figure 2 shows a 3-D view of a silicon sample processed with an increasing number of passes (1,2,5 back and forth passes) at $11 \mu \mathrm{J}$ per pulse, and a translation velocity of $10 \mu \mathrm{m} / \mathrm{s}$. We used a beam accelerating on a circular trajectory of $120 \mu \mathrm{m}$ radius. We observe that curved trenches are processed. Over 5 passes,

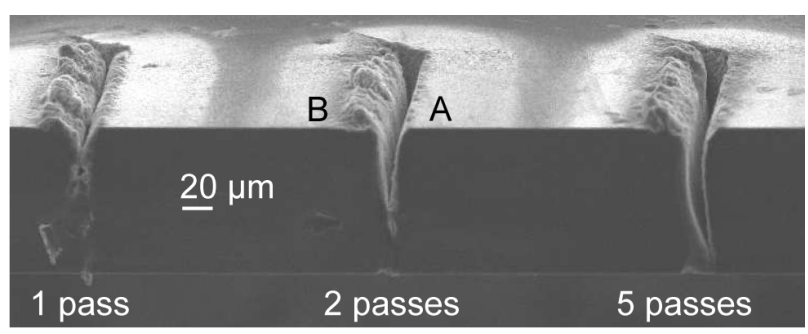

FIG. 23 D view of a silicon sample processed at a sample translation speed of $10 \mu \mathrm{m} / \mathrm{s}$ and a laser energy of $11 \mu$ j per pulse, for an increasing number of back and forth passes.

the drilling of the curved trench saturates. The curvature corresponds to the one of the intense core of the beam, the ablated part is on the side where the subsidiary lobes of the accelerating beam propagate. To help the reader, the letters $A$ and $B$ indicate respectively the side of the intense core trajectory (ie the caustic trajectory), and the side where the subsidiary lobes of the beam propagate.

To investigate the drilling mechanism, we have performed a series of trench processing at varying input pulse energy, and fixed 5 one-way passes. The results are shown in Figure 3. We readily observe that as the pulse energy is increased, the trenches are deeper and progressively follow the beam trajectory that is represented as a white dashed line. Our main objective here is to explain the underlying mechanisms and particularly what stops the drilling. We show that the process rely on the specific structure of the 3D Poynting vector in the circular accelerating beam.

\section{ANALYSIS}

Figure 3(b) shows the beam cross section in $(x, z)$ plane, corresponding to the plane where we observe the trench profiles. As mentioned in the introduction, accelerating beams can be viewed as a specific case of a caustic, where the geometrical rays propagating in the plane $\mathrm{y}=0$ are all tangent to the same curve, i.e. the caustic trajectory. The geometrical rays are tangents to the local Poynting vector, and thus indicate the local propagation direction of energy in the beam.

In Figure 3(b), we have traced the geometrical rays propagating in the $(x, z)$ plane, perpendicular to the sample translation direction. It is apparent that the light generating the intensity maximum of the beam at a distance $z_{0}$ arises from a region $x_{0}$ on the sample entrance surface. Then, during the processing of an opaque sample, the trench is opened at first on top surface where the beam has a maximal intensity, allowing the propagation of a part of the laser beam. We understand that ablation can occur along the caustic at a depth $z_{0}$ if a beamlet can be transmitted from $x_{0}$ on the sample entrance to $z_{0}$ on the caustic. Red lines in Figure 3(a) show the rays linking the extremal ablation side on the caustic trajectory to the entrance surface. For each energy, we have plotted two rays that correspond to the error bar on the measurement of the ablation site position on the caustic location. The error bar is $5 \mu \mathrm{m}$ in average which results in an uncertainty of $\sim 2.5^{\circ}$ on the slope of the rays. 

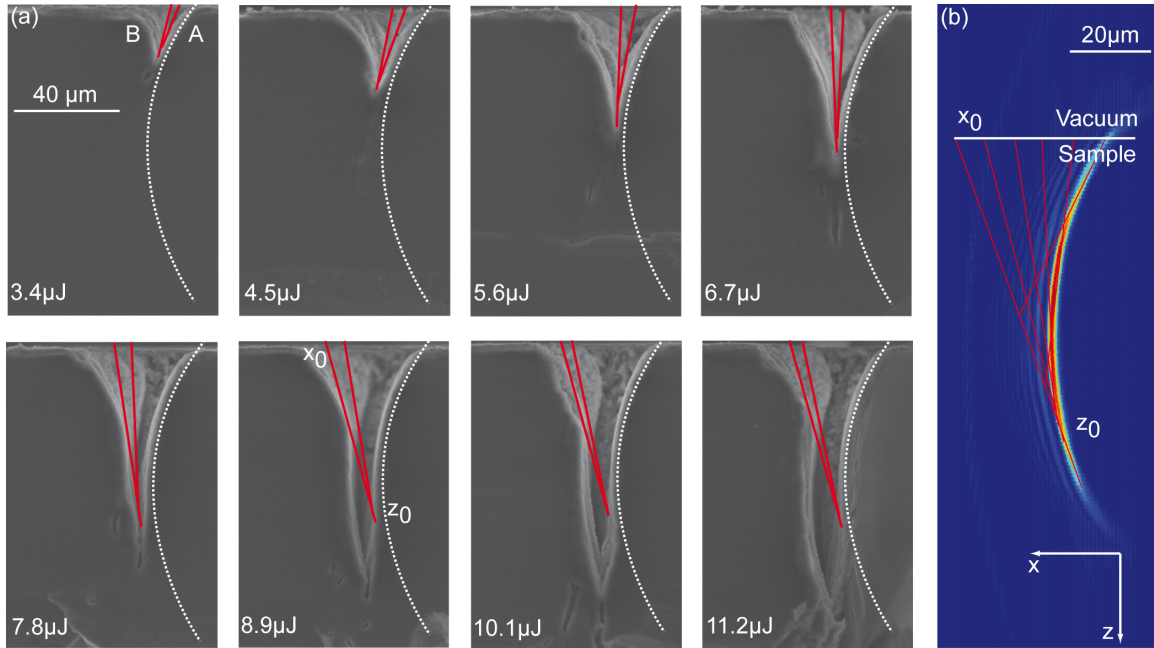

FIG. 3 (a) Profiles of trenches processed at a constant number of 5 passes with increasing pulse energy. The beam trajectory is shown as a white dashed line. Red lines correspond to the optical rays arriving at the maximal trench depth along the circular trajectory (see text). (b) Experimental intensity distribution of the accelerating beam. Red lines show the corresponding geometrical ray structure. The white line indicates the position where the entrance surface of the sample was placed.

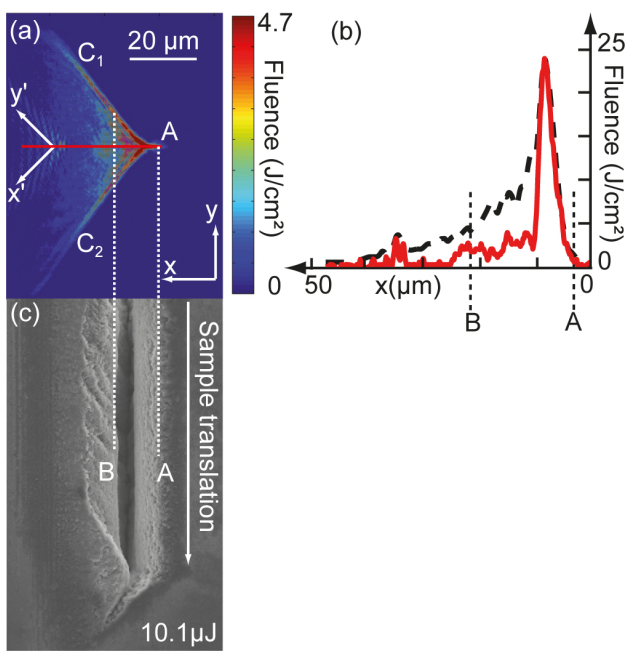

FIG. 4 (a) Experimental beam fluence distribution on the sample entrance surface, saturated at $4.7 \mathrm{~J} / \mathrm{cm}^{2}$, for a pulse energy of $10.1 \mu \mathrm{J}$. (b) The solid red curve shows the fluence profile on the red line of (a), the dashed black line corresponds, for each value of $x$ to the maximal fluence reached on $y$ axis. (c) SEM top view of the silicon sample processed at $10.1 \mu \mathrm{J} /$ pulse. The scale is the same as in (a).

Although this picture allows us to understand the drilling mechanism in depth, it is yet incomplete. Indeed, Figure 4 shows the measured intensity distribution on the sample entrance surface plane $(x, y)$ : the red solid line indicates the origin of the beamlets shown in red in Figure 3(a). In the vicinity of point $A$, the intensity is maximal, but rapidly drops close to zero, as shown in Figure 4(b), solid red line. Therefore, surface ablation and trench opening could not be explained by the intensity of this part of the beam.

On the other hand, we can explain trench opening by surface ablation from the 2 dimensional intensity distribution of the beam on the sample top surface. As can be seen in Figure 4(a), the beam shows two lines of maximal intensity $\left(\mathrm{AC}_{1}\right)$ and $\left(\mathrm{AC}_{2}\right)$ that are parallell to $x^{\prime}$ and $y^{\prime}$ axes. It is those lines that primarily contribute to surface ablation. The maximal value of the fluence reached either on $\left(\mathrm{AC}_{1}\right)$ or $\left(\mathrm{AC}_{2}\right)$ is plotted as a function of $\mathrm{x}$ as a dashed black line in Figure 4(b). The axis was scaled for pulses of $10.1 \mu \mathrm{J}$ and the colorbar was saturated at $4.7 \mathrm{~J} / \mathrm{cm}^{2}$. We note a slight asymmetry: $\left(A C_{1}\right)$ is more intense than $\left(\mathrm{AC}_{2}\right)$ because of residual limiting inhomogeneity of the input beam.

To confirm our hypothesis, for each trench processed in silicon at a given energy per pulse, we have measured the value of the maximal fluence reached on $\left(\mathrm{AC}_{1}\right)$ and $\left(\mathrm{AC}_{2}\right)$ at a position $x_{0}$ retrieved by ray-tracing. In Figure 5 , we have plotted this value as a function of the maximal ablation depth $z_{0}$ along the caustic. We observe that the extremal fluence is approximately constant at $4.7 \mathrm{~J} / \mathrm{cm}^{2}$.

The width of the surface crater opened by the part of the beam over this threshold fluence determines the set of rays that can propagate from $z=0$ to the caustic and contribute to ablation. We also note that the fluence on the caustic is much higher than anywhere else in the beam. Due to the constructive interference and focusing of beamlets, the fluence increases along the trajectory linking $x_{0}$ (edge of a surface crater) to $z_{0}$ (extremal ablation site on the caustic). In Figure 4(c), we show the trench processed in silicon at energy per pulse of $10.1 \mu \mathrm{J}$. White dotted lines are drawn to show the positions of the caustic (A) and of the point of extremal distance $x_{0}$ where the fluence threshold is reached (B). On the right side of line A, the sample is unaffected. Between A and B lines, the curved trench is drilled. On the left side of line B, a shallow ablation crater is observed. We note that it was reported that at low fluence $\left(<1 \mathrm{~J} / \mathrm{cm}^{2}\right)$, the ablation rate of silicon gets much smaller than in the high fluence regime [17]. Although it impacts on the shape of the trench close to the sample entrance surface, shallow ablation has no impact on deep ablation along the caustic.

In Figure 3(a), at energies higher than $7.8 \mu \mathrm{J} /$ pulse, the trenches are deeper than $z_{0}$. This can now be understood from the fact that the beamlets emerging from $x<x_{0}$, can freely propagate in the trench and ablate on the left side of the trench. But the final trench width is approximately constant along the longitudinal $z$ direction because the fluence distri- 


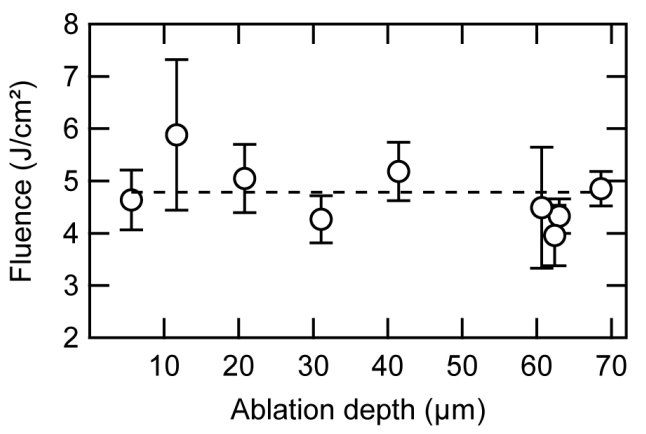

FIG. 5 Maximal fluence reached at the position $x_{0}$ determined for each trench presented in Figure 3 . The results are plotted as a function of the trench depth along the trajectory.

bution follows a circular trajectory and the fluence rapidly drops down when the beamlets departs from the caustic region. The limiting factor to determine the shape of the trench from the beam structure is the existence of reflections and scattering on the sides of the ablated structure, that deviates the impinging beam from its original direction.

\section{CONCLUSION}

As a conclusion, we have investigated material processing with beam accelerating on a circular trajectory and have analyzed the mechanism behind drilling. This mechanism is based on the Poynting vector structure of the beam. Surface ablation was described in terms of fluence threshold and the depth of the trench was determined from the width of this crater. We expect that a similar behavior can be observed for trench machining of transparent materials. In addition, a careful optimization of the beam structure, such as those presented in reference [18] will allow the increase of the dicing efficiency. We anticipate that these results will lead to novel perspectives in terms of laser machining.

\section{ACKN OWLEDGEMENTS}

The authors acknowledge fundings from Région FrancheComté, University of Franche-Comté, and the Agence Nationale de la Recherche contract 2011-BS04-010-01 NANOFLAM.

\section{References}

[1] M. K. Bhuyan, F. Courvoisier, P. A. Lacourt, M. Jacquot, R. Salut, L. Furfaro, and J. M. Dudley, "High aspect ratio nanochannel machining using single shot femtosecond Bessel beams," Appl. Phys. Lett. 97, 081102 (2010).

[2] M. K. Bhuyan, F. Courvoisier, P. A. Lacourt, M. Jacquot, L. Furfaro, M. J. Withford, and J. M. Dudley, "High aspect ratio taper-free microchannel fabrication using femtosecond Bessel beams," opt. Express 18, 566-574 (2010).
[3] G. A. Siviloglou, J. Broky, A. Dogariu, and D. N. Christodoulides, "Observation of accelerating airy beams," Phys. Rev. Lett. 99, 213901 (2007).

[4] J. Baumgartl, M. Mazilu, and K. Dholakia, "Optically mediated particle clearing using Airy wavepackets," Nat. Photonics 2, 675-678 (2008).

[5] P. Polynkin, M. Kolesik, J. V. Moloney, G. A. Siviloglou, and D. N. Christodoulides, "Curved Plasma Channel Ceneration Using Ultraintense Airy Beams," Science 324, 229-232 (2009).

[6] I. Kaminer, R. Bekenstein, J. Nemirovsky, and M. Segev, "Nondiffracting Accelerating Wave Packets of Maxwell's Equations," Phys. Rev. Lett. 108, 163901 (2012).

[7] Y. Kaganovsky, and E. Heyman, "Nonparaxial wave analysis of three-dimensional Airy beams," J. Opt. Soc. Am. A 29, 671-688 (2012).

[8] F. Courvoisier, A. Mathis, L. Froehly, R. Giust, L. Furfaro, P. A. Lacourt, M. Jacquot, and J. M. Dudley, "Sending femtosecond pulses in circles: highly nonparaxial accelerating beams," Opt. Lett. 37, 1736-1738 (2012).

[9] M. Greenfield, E. Segev, W. Walasik, and 0. Raz, "Accelerating Light Beams along Arbitrary Convex Trajectories," Phys. Rev. Lett. 106, 213902 (2011).

[10] L. Froehly, F. Courvoisier, A. Mathis, M. Jacquot, L. Furfaro, R. Giust, P. A. Lacourt, and J. M. Dudley, "Arbitrary accelerating micronscale caustic beams in two and three dimensions," Opt. Express 19, 16455-16465 (2011).

[11] P. Aleahmad, M. A. Miri, M. S. Mills, I. Kaminer, M. Segev, and D. N. Christodoulides, "Fully Vectorial Accelerating Diffraction-Free Helmholtz Beams," Phys. Rev. Lett. 109, 203902 (2012).

[12] P. Zhang, Y. Hu, T. Li, D. Cannan, X. Yin, R. Morandotti, Z. Chen, and $X$. Zhang, "Nonparaxial Mathieu and Weber Accelerating Beams," Phys. Rev. Lett. 109, 193901 (2012).

[13] M. A. Alonso, and M. A. Bandres, "Spherical fields as nonparaxial accelerating waves," Opt. Lett. 37(24), 5175-5177 (2012).

[14] A. Mathis, F. Courvoisier, L. Froehly, L. Furfaro, M. Jacquot, P. A. Lacourt, and J. M. Dudley, "Micromachining along a curve: Femtosecond laser micromachining of curved profiles in diamond and silicon using accelerating beams," Appl. Phys. Lett. 101, 071110 (2012).

[15] I. D. Chremmos, Z. Chen, D. N. Christodoulides, and N. K. Efremidis, "Abruptly autofocusing and autodefocusing optical beams with arbitrary caustics," Phys. Rev. A. 85, 023828 (2012).

[16] F. Courvoisier, P. A. Lacourt, M. Jacquot, M. K. Bhuyan, L. Furfaro, and J. M. Dudley, "Surface nanoprocessing with nondiffracting femtosecond Bessel beams," Opt. Lett. 34, 3163-3165 (2009).

[17] T. H. R. Crawford, A. Borowiec, and H. K. Haugen, "Femtosecond laser micromachining of grooves in silicon with $800 \mathrm{~nm}$ pulses," Appl. Phys. A 80, 1717-1724 (2005).

[18] M. A. Bandres “Accelerating beams," Opt. Lett. 34(24), 3791-3793 (2009). 